\title{
About the influence of hardening conditions on the structure and properties of expansive concretes
}

\author{
Vyacheslav Alekseev ${ }^{1}$, Igor Harchenko ${ }^{1}$, Aleksey Harchenko ${ }^{2}$, and Sofia Bazhenova ${ }^{l}$ \\ ${ }^{1}$ Moscow State University of Civil Engineering, Yaroslavskoe shosse, 26, Moscow, 129337, Russia \\ ${ }^{2}$ Production Company «InGeoStroy», 109147, Moscow, Russia
}

\begin{abstract}
The paper describes the effect of hardening conditions on the hydration of cement stone and methods of controlling the concrete formation on the basis of the above research methodology. It has been proved that when the humidity of the medium in which hardening takes place is regulated, the strength and the deformation of the material change. By adding an expanding component to concrete, it is possible to adjust the technical parameters of concrete and simulate the durability of concrete structures. Thus, the study of the mechanisms of volumetric deformation during hardening of cement stone with a different ratio of the expanding additive allows designing concrete compositions for different operating conditions.
\end{abstract}

\section{Introduction}

At the present time in the cast-in-place construction practice different composite binders are extensively used that allow production of concretes with pre-determined properties, with due account of the variety of design requirements applied to the quality of modern concrete and reinforced concrete structures.

Herewith, the task related to the necessity of reduction of shrinkage strain impact on the concrete properties and the quality of erected structures is in the most cases the most important and crucial one, as the shrinkage cracks are the main source of the development of corrosion of concrete and reinforcement, seepage, reduction or total loss of serviceability of structures.

One of the effective methods to eliminate shrinkage impact and increase crack resistance of concrete and reinforced concrete structures is the application for their production of expansive cements (EC), which are often erroneously classified as shrink-proof ones [1-4].

Misconception of expansive cements as of shrink-proof concretes is one of the reasons that, despite of more than hundred-year history of development, they find rather limited use in constructional practice. The essence of erroneous idea is that at their air hardening moist shrinkage, enhanced by the structure carbonization under influence of $\mathrm{CO} 2$ in the air, manifests itself to the full extent. (Fig.1) 


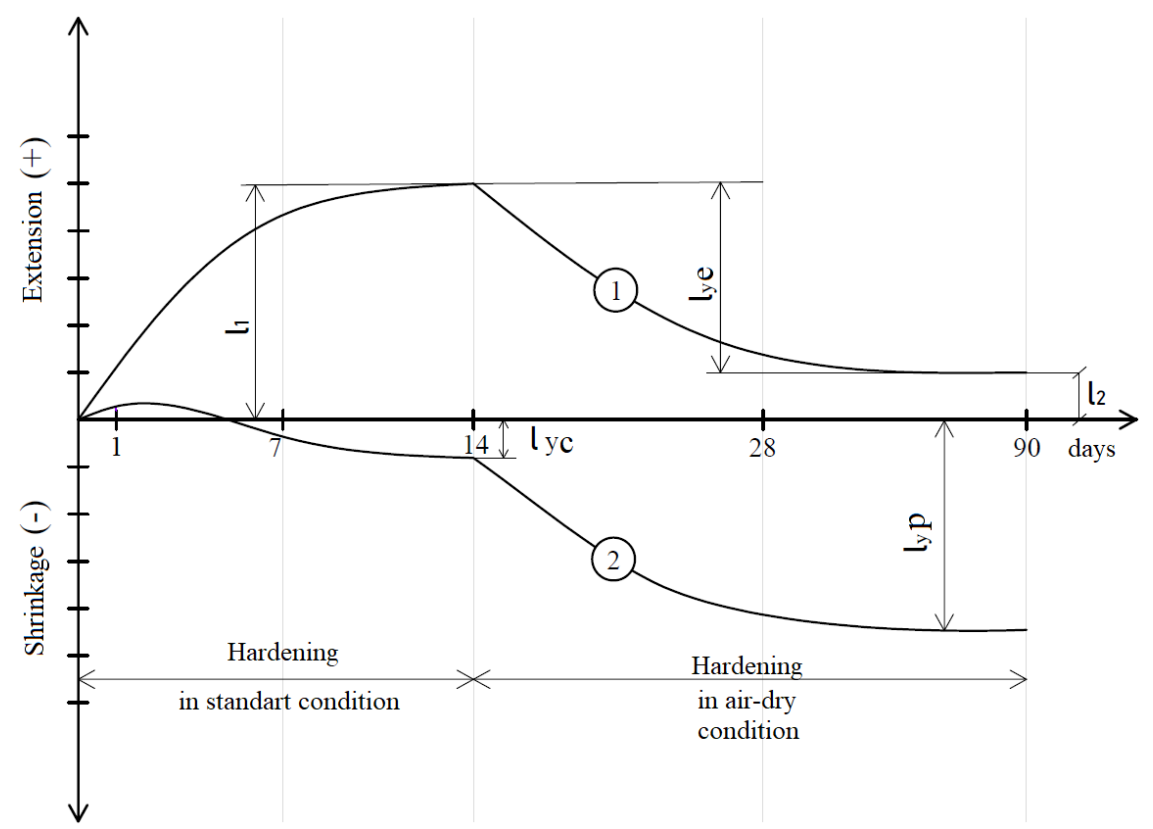

Fig 1. Kinetics of deformations during hardening of standart Portland cement and expanding cement: 11-extension,; ly- contraction shrinkage; lye - desiccation shrinkage of expanding cement; lyp - desiccation shrinkage of Portland cement; 12 - permanent expansion.

Herewith, absolute values of shrinkage both for normal concrete and for expansive concrete are commensurable [6,7]. Fundamental difference is in the value of after-expansion after stabilization of all types of shrinkage appeared. However, crack resistance of structures on the EC basis is, as a rule, substantially higher than the crack resistance of normal concretes, as the values of tensile stress in its structure are lower or completely absent. In this case concrete is in the constricted condition, e.g., at application of one of EC types known as self-stressing cement $[3,14]$.

It is known that the properties of hardened expansive cements and concretes on their basis are to a great extent defined with the degree of consistency of concurrently developing processes: strengthening of Portland cement matrix and its expansion due to ettringite crystallization. At the initial stage of hardening, which usually develops during the period of up to 7 days, it is necessary to provide the optimum kinetic correspondence between the gain in strength of Portland cement matrix and ettringite formation due to expansive additive hydration. Analysis of the results of multi-year research and operational experience in different types of EC demonstrates that in early age, during the period of up to 14 days, the structure of hardening cement stone with the expansive additive is more sensitive to temperature and humidity conditions of hardening than at application of normal construction cements.

During the period of structure stabilization, which usually lasts up to $14 \div 28$ days, basic strength and stress-related characteristics of the cement stone and concrete are formed, formation of its porous structure ends, which to a great degree stipulates achieving of design requirements to the expansive concrete and building structures on its basis. In view of the foregoing considerations, the objective of the present paper is the investigation of influence of watering conditions on the development of processes of forming of structure and properties of expansive cement and concrete on its basis.

\section{Applied materials and research methods}


As far as ettringite is a major structure-forming element of the EC, there was conducted a research of the influence of different temperature and humidity conditions on its stability with due account of morphological features of its crystals structure. The research was conducted applying a complex of physical and chemical methods, such as X-ray phase analysis, infrared spectroscopic analysis and differential thermal analysis completed with the research applying scanning electron microscope with the magnification from 1:3000 to 1:10000.

Ettringite crystals were synthesized by means of mixing of water solutions of hydrated lime and aluminum sulfate in stoichiometric ratio necessary for ettringite formation. Specified value of $\mathrm{pH}$ in the reaction solution was set by means of introduction to its composition of citric acid or atropine alkali being an alkaloid alkali of organic nature. The value of $\mathrm{pH}$ was determined by means of potentiometric method using combination solid-contact electrodes of EST type with the accuracy of $0,05 \ldots 0,1$. Ettringite crystallization took place at $\mathrm{T}=20 * \mathrm{C}$ with further filtering and preservation of the sediments. For quantitative assessment of formed ettringite crystals the correlation between the longitudinal dimension and transverse dimension of the crystal was used [6].

During investigation of the influence of carbonization on the ettringite stability it was assumed that under normal conditions the air almost constantly contains $0,03 \%$ of carbon dioxide, which corresponds to $600 \mathrm{mg} / \mathrm{m}^{3}$ [7].

In order to accelerate carbonization process an ettringite stability investigation was carried out at elevated concentration equal to $0,1 \%$ at relative humidity of $70 \%$, which corresponds to carbon dioxide content in stock buildings, garages or road tunnels [14]. In order to investigate the influence of hardening conditions on the structure and properties of ECs, expansive cements with different content of expansive additive were used, including ECs with extremely high expansion potential of up to $10 \%$, which were obtained by mixing basic low aluminate Portland cement TsEM 42,5 with the expansive additive on sulfoaluminate basis in the quantity of 5,15 and $25 \%$.

Expansive (expanding) additive was prepared on the basis of high-alumina cement «Secar $70 »$ and calcium sulfate dehydrate. Investigation was carried out for sand-cement mortar with the composition 1:2 with constant fluidity with cone flow diameter of $200 \mathrm{~mm}$. According to the research program, after stripping at the age of 1 day samples hardened prior to testing at the age of 28 days after hydraulic setting with further dry curing during 28 days (series No.4); at $100 \%$ relative humidity during 14 days with further hardening during 28 days in the air (series 3); 3 days in water with further dry curing for 28 days (series 2).

Strength of fine-grained concrete was determined in compliance with the GOST 307442001 «Cements. Testing methods using multifractional sand». Pore structure was investigated by means of mercury porosimetry method and scanning electron microscope, freezethaw resistance was determined by the sample weight loss at cyclic freezing and thawing, hydration products were studied by means of X-ray phase analysis.

\section{Investigation results}

Based on the analysis of the results of the research of stability of synthetic ettringite at storing under dry air conditions $\left(20^{\circ} \mathrm{C}, 65 \%\right.$ relative humidity) with different $\mathrm{CO} 2$ concentration it was established that the ettringite that was produced at the $\mathrm{pH}$ of greater than 12,5 after curing in the air with elevated content of carbon dioxide during 2 years remains in stable condition [6].

Synthetic ettringite that has crystallized at lower $\mathrm{pH}(10 \div 11,5)$ destructed in as little as 48 hours at storing in the air. Thus, stability of ettringite as of a component of expansive cement structure to a great extent depends on the morphology of its crystals that was formed under the influence of the reaction solution $\mathrm{pH}$. 
At obtaining of expansive cement by means of mixing of finished Portland cement with expansive components on sulfoaluminate basis ettringite is formed in a strong alkaline environment mainly in the form of crystal needles with length/diameter ratio of $18 \div 22$. At introduction to the composition of expansive cement of alkaline components increasing $\mathrm{pH}$ to 13 and higher, ettringite is formed in a dense crystalline form with $\mathrm{L} / \mathrm{D}=1 \div 3$ (Fig. 2).

a)

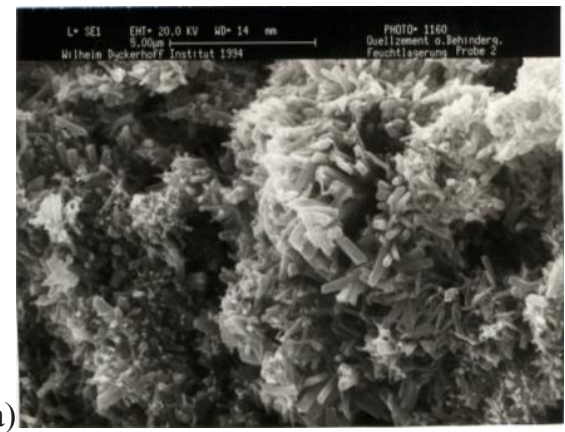

b)

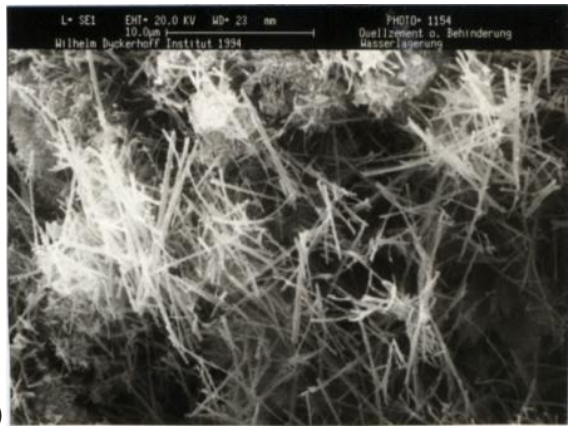

Fig 2. Effect of $\mathrm{pH}$ on the morphology of ettringite crystals: $\mathrm{pH}=13,0$ (a) и $\mathrm{pH}=11,5$ (b)

Ettringite with this morphology possesses the highest stability at different storage conditions including reduced humidity up to $30 \%$ and elevated temperature of up to $80^{*} \mathrm{C}$, which simulates the operating conditions for enclosing parts under conditions of intense solar radiation.

Under the influence of carbon dioxide contained in the air the value of $\mathrm{pH}$ in pore structure of cement stone and concrete reduces from its initial value of 12,6 .13,0 to 9 and less, which is accompanied by hydrated lime transformation into calcite, as well as changing of other hydration products. Herewith, at Portlandite transition into calcite the volume increase of up to $11 \%$ is observed, which additionally stresses the cement stone structure [14].

Shrinkage strain developing under carbonization influence is primarily the consequence of evaporation of the water released at dehydration of calcium hydroxides $[8,9]$.

Comparative analysis of kinetics of structure formation and properties of EC with high expansion potential of up to $100 \mathrm{~mm} / \mathrm{lin} . \mathrm{m}$ that harden at different temperature and humidity conditions revealed that for EC hardening in water with further dry curing the formation of dense network of shrinkage cracks over the surface is characteristic. This may be explained with the following: at hardening of expansive concretes the process of creation of inherent stress fields develops in more complicated manner than for concretes on the basis of normal Portland cement.

Thus, if at formation of the structure of cement stone on Portland cement basis tensile stresses along the specimen outline are formed only under influence of carbonization water loss, then at hardening of expansive cements the gradient of inherent stresses is substantially increased due to the development of expansion process in internal areas and shrinkage in external areas at drying and carbonization. Besides, due to formation of fine-pored structure of cement stone of expansive cements humidity gradient between the inner and outer areas of cross section increases. This is followed by forming of the network of cracks with the depth of up to $1 \ldots 3 \mathrm{~mm}$ over the surface of cement stone and concrete, which may become corrosion centers for concrete and reinforcement at further operation. Analysis of the results of investigation of structure and properties of expansive cement stone placed in dry-air conditions in early age (Fig.3, specimen 2) demonstrated that its upper layer contains substantial amount of calcite, monosulfate, Portlandite and CSH-phases at almost complete absence of ettringite. Herewith, the strength of outer layers is $10 \ldots 15 \%$ lower than the strength of inner ones, and cracks with the depth of up to $1 . . .3 \mathrm{~mm}$ are formed over the surface. Internal area 
of hardened expansive cement contains CSH-phase, Portlandite and ettringite, and its microstructure is free from cracks and has high strength and density.

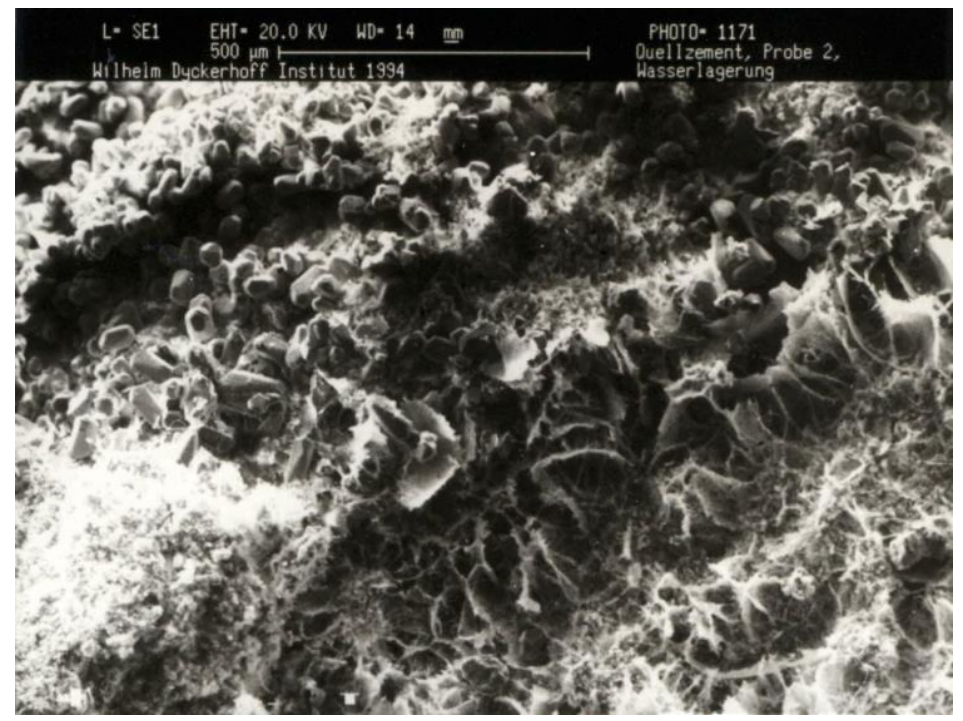

Fig. 3 Structure of the peripheral zone of fine-grained concrete with expanding additive after aging in air-dry conditions.

Analysis of the results of experimental research demonstrated that in the case of provision of concrete on the EC basis thorough curing during the period of the most active hardening of up to $7 \ldots 14$ days by means of creation of optimum temperature and humidity conditions it is possible to obtain concrete with high strength, crack resistance, water and gas tightness and durability. Herewith, one of the most sensitive indices of durability of expansive cements and concretes on their basis is the sulfate resistance, which mainly depends on total quantity of calcium aluminates that are contained in clinker and expansive additive.

Herewith, if the quantity of calcium aluminates contained in expansive additive is set on the basis of the condition of optimum stoichiometric correlation with calcium sulfate, which is necessary for completion of binding in ettringite during the period of active structure formation, then binding of calcium aluminates in clinker composition is hard to predict. Accordingly, at designing of compositions of EC intended for operation under conditions with elevated concentration of sulfate ions, it is recommended to apply low aluminate Portland cements as Portland cement matrix.

In order to implement all potential positive properties of expansive cements, it is necessary not only completely bind calcium aluminates during the period, when the matrix structure is in elastic-plastic condition and is capable of deforming without cracking, but also create optimum temperature and humidity conditions of hardening excluding development of inherent stress gradient across the cross-section of hardening concrete. It means that at practical application of expansive cements and concretes on their basis it is necessary to bear in mind that at early stage of hardening their structure is more sensitive and requires prevention of water loss and thorough curing after completion of concreting.

If otherwise, the risk of crack formation over the surface of concrete and reinforced concrete structures is substantially higher than at application of normal construction cements. Moist storage and thorough curing at hardening of expansive concretes must be provided at least during the period of up to 7 days after completion of concreting, until the strength of Portland cement matrix reaches the value necessary to take tensile stress occurring at the development of internal stress. (Fig.4). 


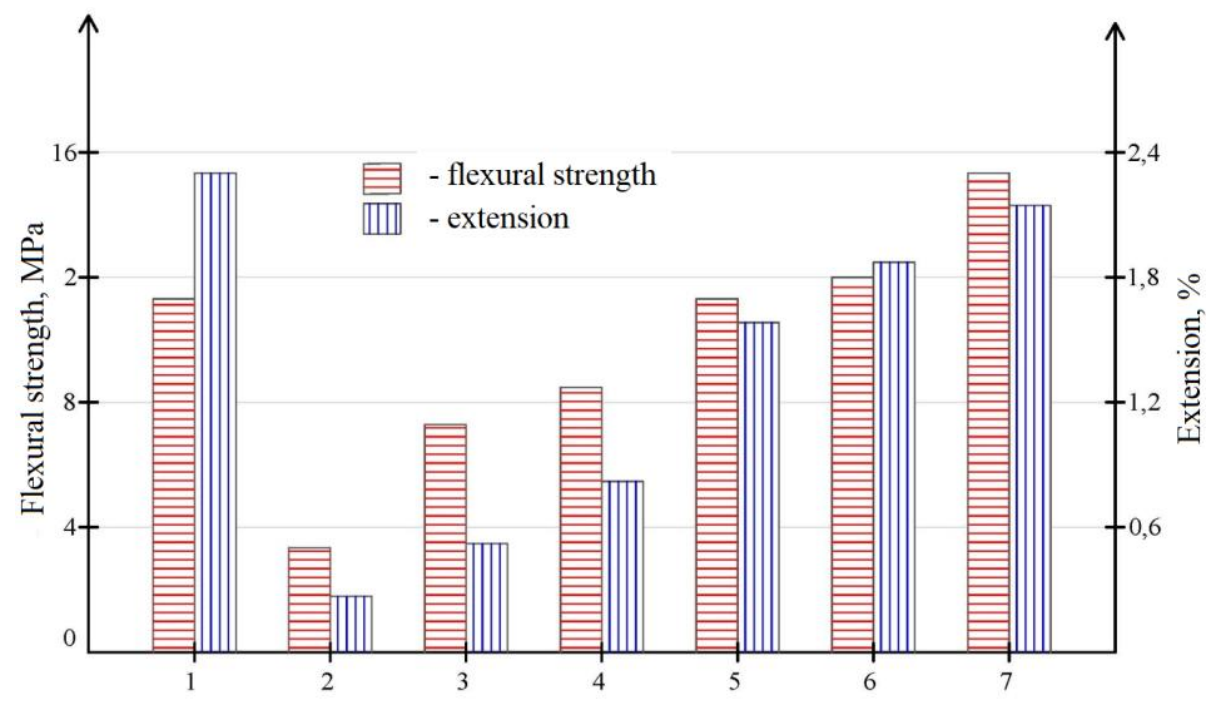

Fig. 4. Influence of conditions of leaving on durability and expansion of fine-grained concrete: $1-$ hardening in water; $2 \ldots 7$ - hardening in air-dry conditions with strengths in $\%$ of branded $(100 \%)$, respectively: $2-10 \% ; 3-20 \% ; 4-35 \% ; 5-50 \% ; 6-75 \% ; 7-100 \%$

A large body of research of the process of forming of structure of expansive cements at relative humidity of about $100 \%$ or at storing in water demonstrated that the kinetics of expansion process, the degree of hydration of Portland cement matrix, the quantity of formed ettringite, and the achievable value of expansion are in both cases commensurable (Fig. 5).

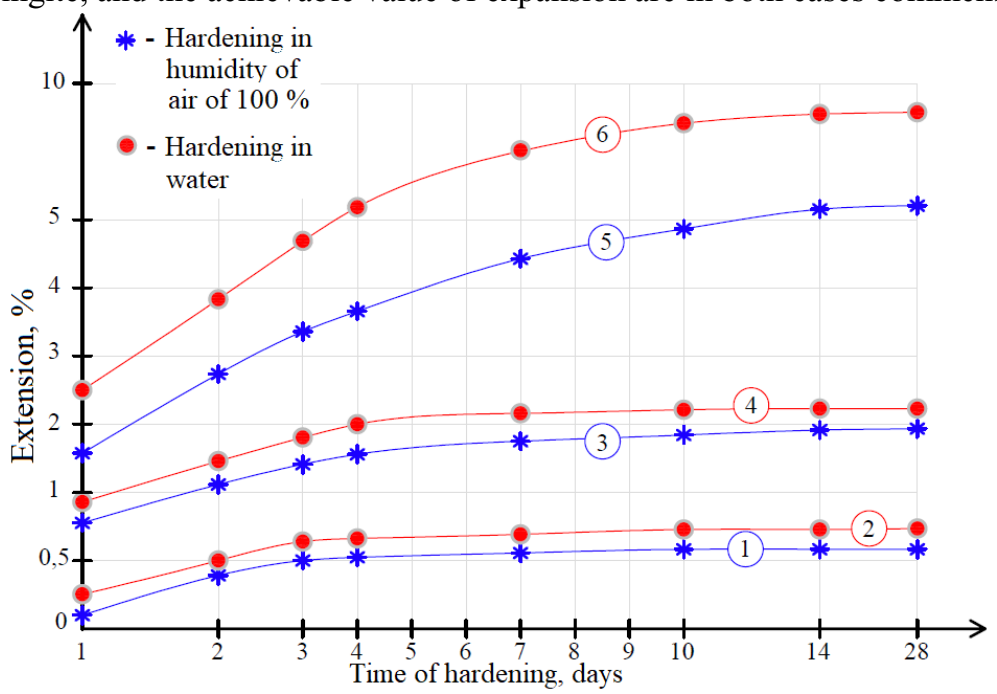

Fig. 5. The expansion kinetics of fine-grained concrete with different contents of the expanding additive ( $1,2-5 \%$ additive; $3,4-15 \%$ additive; $5,6-25 \%$ additive)

It means that the quantity of mixing water is sufficient for complete implementation of expansion potential at hardening. Samples of expansive cement at hydraulic setting had sufficiently dense network of cracks over the surface with opening width of up to $0,5 \mathrm{~mm}$. Samples of the same expansive concrete at storage under conditions of $100 \%$ relative humidity, 
on contrary, even at the extreme value of expansion of up to $10 \%$, have no cracks, though in general their structure is relatively loose and has low density and strength.

Analysis of hydration products for specimens hardening at different conditions revealed that at storing of EC specimens in water fine-crystalline fibrous ettringite is formed with $\mathrm{L} / \mathrm{d}$ ratio $=40 \ldots 60$. At moist storage ettringite is crystallized in classical form, as relatively short and thick hexagonal needles with $\mathrm{L} / \mathrm{d}=15 \ldots 20$. These essential differences in the morphology of formed ettringite may be explained with different ion content in pore solution and especially with different $\mathrm{pH}$ value in the reaction medium [6].

At storage in water under influence of concentration gradient calcium hydroxide is partially washed from the structure of cement stone contacting with water, which leads to the reduction of $\mathrm{Ca}$ - concentration, and primarily, of $\mathrm{OH}$-ions concentration. This is followed by the formation of fine-fibred ettringite with $\mathrm{L} / \mathrm{d}=40$ and more.

According to the conducted research, ettringite with this morphology at dry-air storage $\left(20^{\circ} \mathrm{C}, 65 \%\right.$ relative humidity), is in unstable condition and quickly recrystallizes into monosulfate, which is followed by the reduction in strength of cement stone and concrete. This explains the formation of cracks at storage in water of concretes on expansive cements with high expansion potential of up to $10 \%$, when the contact layer of expansive cement stone became loose under influence of capillary seepage followed by the development of inherent stresses in the cross-section.

Besides, calcium hydroxide is dissolved and washed from the pore space thus reducing the $\mathrm{pH}$ value, which results in the formation of fine-crystalline fibrous ettringite followed by loosening of structure of cement stone in the contact area. Thus, at storage in water, the developing expansion process is accompanied by the tensile stressing related to the formation of loose ettringite due to capillary seepage in the area of contact with the water medium. This leads to creation of additional stress in the concrete outer layers that manifests itself in the formation of crack dense network over the surface. It is worth noting that the formation of surface cracks is characteristic in the cases of application of expansive cements with extremely high expansion potential of up to $10 \%$.

However, the described process of features of formation of structure of cement stone on expansive cements at storage in water is also characteristic for all other expansive cements, including EC $\mathrm{c}$ with low expansion potential of up to $1 \%$. In view of the foregoing we can make the following important conclusions accounting for the technology distinctions for concretes on expansive cements: for implementation of all potential possibilities of expansive cements and for provision of complete development of expansion process it is unnecessary to store them in water. It is entirely sufficient to provide moist storage excluding losses of mixing water during the period of up to 14 days.

Due to this at hydration of expansive cements a crack-free structure of cement stone is formed independently of the value of expansion potential. Storage in water is feasible only for substantial content of expansive additive of more than $15 \%$, substantial expansion potential and mandatory plane or volumetric restriction of expansion strain.

In the construction practice at application of expansive cements free unbounded increase in volume usually manifests itself only in outer protective layer of reinforced concrete structures.

Increase in inner volume of concrete and reinforced concrete structures is restricted by the reinforcement, formwork or adjacent structures in butt joints. In this connection the cross section of construction elements, as a rule, contains tensile stress gradient, which grows due to water loss and corresponding shrinkage, which may be followed by the formation of cracks over the surface of structures. In order to prevent this during the development of process regulations at application of concretes on the EC basis it is necessary to tend to reduce the difference between the expansion value at free hardening and at restricted expansion. This 
goal is achieved, e.g., at application of expansive cements with low expansion potential of up to $3 \%$ and long-term (not less than 14 days) hardening under moist conditions.

As was already mentioned, at operation of building structures on the basis of expansive cements in the air there occur not only physical changes in structure of concrete due to appearing of humidity gradient, but also are observed the changes in hydration products under action of carbon dioxide present in the air. Herewith, calcium hydroxide affected by $\mathrm{CO}_{2}$ transforms into calcium carbonate, which leads to the development of so-called carbonaceous shrinkage that must be accounted in total volume of shrinkage strain. Despite of the fact that the share of carbonaceous shrinkage does not exceed $20 \%$ of total shrinkage volume for expansive concretes, an important element of which structure is ettringite, the affect of carbon dioxide causes more essential influence. Due to recrystallization of calcium hydroxide and formation of calcite the $\mathrm{pH}$ value in pore solution decreases, which is followed by ettringite decomposition with forming of monosulfate $[4,5]$.

On the basis of analysis of the results of experimental research it is established that at increase of curing period from 3 to 7 days, carbonization depth reduces from 8 to $3 \mathrm{~mm}$, and at curing period of up to 14 days it does not exceed $1,5 \ldots 2 \mathrm{~mm}$. Herewith, the share of open capillary pores in the total volume of pore space is substantially reduced from $36 \%$ to $12 \%$, respectively, with predominant content of pores from 10 to $300 \mathrm{~nm}$. Thus, for provision of pre-determined quality of concretes on expansive cement basis it is extremely important to organize qualitative curing following the concreting.

The research of durability of prefabricated and monolithic reinforced concrete envelopes (external wall panels, structures of uncovered reinforced concrete roofs) produced on the basis of self-stressing cements that were operated for a long time in different natural climatic conditions demonstrated that due to the development of shrinkage strain the loss in value of self-stressing does not exceed 55\% at maintaining of design strength. $/ 24 /$ Herewith, a preserved level of self-stressing satisfied design requirements to crack resistance, and figures for strength, water tightness and freeze-thaw resistance were essentially higher than for normal concretes on the Portland cement basis.

\section{Conclusions}

It is established that hardening of expansive concretes in early age during the period of up to $7 \ldots 14$ days under dry air conditions is followed by the intense cracking due to the development of different types of shrinkage strain. It means that the expansive concretes are more sensitive to curing conditions than normal concretes. For obtaining of expansive concretes with elevated crack resistance and implementation of all potential possibilities of expansive cements it is sufficient to provide exclusion of water losses during the period of not less than 14 days instead of storage in water.

\section{References}

1. E. Candlot, E. Arts chimiques. Rapport de Le Chatelier sur un memoire de M. Candlot ayant trait aux propietes des ciments. (Bull.Soc.Encour) (1990)

2. W. Michaelis, Der Zement (Bazillus, TonindZtg, 1892):

3. T. Kuznetcova Aluminate and sulphoaluminous cement (Moscow, Stroyizdat, 1986).

4. L. Xu, et. al. Construction and Building Materials, 31 347-352 (2010)

5. I.J. Harchenko, Arch.Bauwes. Weimar 39, p. 171-177, (1993).

6. I.J. Harchenko 10th Intern. Coal Ash Symposium, (Florida/USA), p. 6-14 (1993).

7. W. Li, M. Fall. Construction and Building Materials, 106, 296-304 (2016). 
8. J. Stark, Cement and lime (Kiev, 2008).

9. A. Chen, W. Hargis, C.G. Juenger. Cement and Concrete Research, 42, 1, .51-60 (2012)

10. J.L. García D. Revuelta, J.J. Sánchez, J.P. Gutiérrez. Construction and Building Materials, 93, 223-229 (2015).

11. T., Lahajnar G. Sepe, A. Blinc et. al. Cement and Concrete Research, 31, 2, 263-269 (2001).

12. N. Cefis, C. Comi. Cement and Concrete Research, 93, 57-70 (2017).

13. V. Rahhal, R. Talero. Construction and Building Materials, 53, p. 674-679, (2014)

14. I. Galan, H. Beltagui, M. García-Maté, F.P. Glasser, M.S. Imbabi. Cement and Concrete Research, 84, 85-94 (2016) 